\title{
Development of a Voltammetric Method to Determine Molybdenum in Food Grains
}

\author{
A.L. Alvarado-Gámez ${ }^{1,2, *}$ C. León-Rojas, ${ }^{2}$ E.R. Espinoza-Castellón ${ }^{1,2}$ \\ ${ }^{1}$ Centro de Electroquímica y Energía Química, ${ }^{2}$ Escuela de Química,Universidad de Costa \\ Rica, Apdo. 2060, San Pedro de Montes de Oca, San José, Costa Rica.
}

Received 31 May 2004; accepted in revised form 22 September 2005

\begin{abstract}
It was developed a very sensitive analytical method to determine molybdenum in water and food grains, using the linear sweep voltammetry technique, with adsorptive preconcentration of a Mo(VI) 8-hydroxyquinoline complex, in an acetate buffer media of $\mathrm{pH}=3.5$ and $\mathrm{KClO}_{3} 0.5 \mathrm{~mol} / \mathrm{L}$ as a support electrolyte. The linear range of the methodology goes from $1.0 \eta \mathrm{g} / \mathrm{L}$ to $10 \mu \mathrm{g} / \mathrm{L}$, with a limit of detection of $0.3 \eta \mathrm{g} / \mathrm{L}$ and quantification of $1.0 \eta \mathrm{g} / \mathrm{L}$. The methodology is suitable in the range of $\mu \mathrm{g} / \mathrm{L}$ and $\eta \mathrm{g} / \mathrm{L}$. The method was applied to grinded samples of rice, beans, lentil seeds and chickpeas with the following result: $6.2 \mathrm{mg} / \mathrm{kg}$ in lentil seeds, $6.0 \mathrm{mg} / \mathrm{kg}$ in chickpeas, $3.2 \mathrm{mg} / \mathrm{kg}$ in red beans, and $1.7 \mathrm{mg} / \mathrm{kg}$ in rice.
\end{abstract}

Keywords: linear sweep voltammetry, complexes, trace analysis, molybdenum, 8hydroxyquinoline, adsorptive preconcentration.

\section{Introducción}

El molibdeno es un elemento traza esencial tanto para el ser humano como para las plantas, los animales y los microorganismos. Se sabe que algunas plantas tienen problemas de floración y de producción de polen cuando hay deficiencia de este elemento. Por el contrario, niveles altos en el suelo, generan un crecimiento anormal de algunas plantas. En los procesos de auto purificación de aguas naturales, niveles del orden de $5 \mathrm{mg} / \mathrm{L}$ son suficientes para inhibir el crecimiento de microorganismos deseables [1,2].

\footnotetext{
* Corresponding author. E-mail address: agamez@cariari.ucr.ac.cr
} 
El molibdeno es esencial para algunas enzimas que actúan en el metabolismo de plantas y animales, por ejemplo para la mamalia xantina oxidasa y xantina deshidrogenasa, la aldehído oxidasa, sulfito oxidasa, formato deshidrogenasa, nitrato reductasa y nitrogenasa. Tiene además una función de transporte de electrones en las enzimas que catalizan la reducción de nitrógeno a nitrato. En las plantas se hace necesario también para la reproducción aún cuando está presente en los tejidos a niveles más bajos que $0.5 \mathrm{mg} / \mathrm{kg}$ en base seca, más que los niveles críticos de otros elementos esenciales.

En el caso de los seres humanos es necesario al menos para tres enzimas. La enzima sulfito oxidasa cataliza la oxidación del sulfito a sulfato, necesario para el metabolismo de aminoácidos con azufre. La deficiencia o ausencia de esta enzima lleva a síntomas neurológicos y muerte temprana. La xantina oxidasa cataliza la hidroxilación oxidativa de purinas y piridinas incluyendo la conversión de hipoxantina a xantina y xantina a ácido úrico. La aldehído oxidasa oxida purinas, pirimidinas, piperidinas y está involucrada en el metabolismo del ácido nicotínico. Una dieta baja en molibdeno lleva a niveles muy bajos de ácido úrico en orina y suero y una excreción excesiva de xantina [3].

El molibdeno se presenta en su forma hexavalente $\mathrm{Mo}(\mathrm{VI})$ en aguas naturales, suelo y plantas, a niveles de concentración de ultratrazas, en el orden de los $\mu \mathrm{g} / \mathrm{L}$, por lo que se hace necesario el establecimiento de metodologías apropiadas de alta sensibilidad y selectividad, para la detección y cuantificación de este elemento a esos niveles de concentración.

Las técnicas voltamperométricas son muy adecuadas para la determinación de metales como $\mathrm{Pb}, \mathrm{Cd}, \mathrm{Zn}$ y otros formando amalgamas en una gota de mercurio y de metales de transición como por ejemplo $\mathrm{Cr}, \mathrm{Fe}, \mathrm{Ni}, \mathrm{Co}, \mathrm{Mo}$, por la técnica de preconcentración adsortiva de complejos formados con ciertos ligandos, que se oxidan o reducen al ser adsorbidos sobre la gota suspendida de mercurio, muchas veces con ayuda de agentes catalíticos.

En la literatura, algunos autores han utilizado estas técnicas para la determinación de Mo y se mencionan agentes acomplejantes tales como la 8hidroxiquinoleína [1,4], el ácido mandélico [2], la tropolona [1,5], el ácido cloroanílico [5-7], la $\alpha$ benzoinoxima [8] y el 2-(2`-thiazolylazo)-p-cresol (TAC) [9], lo mismo que el anaranjado de metilo [10]. También se menciona el uso de $\mathrm{NaNO}_{3}$ y el $\mathrm{NaClO}_{3}$ como catalíticos, $\mathrm{KNO}_{3}$ como agente oxidante o nitratos y fosfatos como electrolito soporte [1-8,11-13].

\section{Parte experimental}

Para limpiar la cristalería utilizada en el desarrollo del método y su aplicación en muestras reales fue necesario lavarla con un detergente libre de metales, luego enjuagarla con agua destilada y colocarla en baños de $\mathrm{HNO}_{3}$ calidad para análisis al $20 \% \mathrm{v} / \mathrm{v}$, por 2 semanas. El agua para preparar los reactivos y hacer diluciones provenía de un sistema desionizador Barnstead y un purificador Milli Q. El equipo utilizado es un analizador Park 384B y un sistema de electrodos EG\&G, con un microelectrodo de gota suspendida de mercurio como electrodo de trabajo, un electrodo de alambre de Pt como electrodo auxiliar y un electrodo 
de referencia de $\mathrm{Ag} / \mathrm{AgCl}$. Se utilizó la técnica de adición estándar para la cuantificación, utilizando disoluciones patrón de Mo(VI) preparadas a partir de una madre de $1046 \mathrm{mg} / \mathrm{L}$, proveniente de un titrisol Merck. El ácido acético y el acetato de sodio para la preparación del buffer, fueron de calidad ultrapura y el $\mathrm{KClO}_{3}$ y la 8 hidroxiquinoleína, de calidad para análisis. El nitrógeno para desoxigenar el sistema es puro y se hizo pasar por una trampa de vanadio para eliminar las trazas de oxígeno.

Se optimizaron varios parámetros para determinar el electrolito soporte adecuado para el análisis de Mo-8 hidroxiquinoleína, tales como: el efecto de la concentración de $\mathrm{KClO}_{3}$ sobre la señal de $\mathrm{Mo}(\mathrm{VI})$, el efecto del $\mathrm{pH}$ sobre la corriente de pico, la variación de la altura de pico y corrimiento de la señal para Mo(VI) a diferentes pHs. Se inició con el método recomendado por Gao y Siong [1]. Se varió solo un parámetro a la vez y se escogió el valor que diera mejor señal de corriente. Además se construyó la curva de electrocapilaridad. Se determinó el mejor potencial de acondicionamiento y su efecto sobre el potencial de pico. Se cuantificó por adición estándar con un patrón de $20 \mu \mathrm{g} / \mathrm{L}$, haciendo adiciones de $40 \eta \mathrm{g} / \mathrm{L}$. Se calculó el límite de detección siguiendo el método de Taylor [14], se analizaron siete blancos independientes y se cuantificaron por adición estándar, se calculó la desviación estándar, se multiplicó por tres y se dividió entre la pendiente.

Se analizaron muestras de granos como arroz, frijoles rojos, lentejas y garbanzos. Todos los granos se liofilizaron y se digirieron con $\mathrm{HNO}_{3}, \mathrm{H}_{2} \mathrm{SO}_{4}$ y $\mathrm{HClO}_{4}$.

\section{Resultados y discusión}

Se estudiaron inicialmente varios electrolitos y su efecto sobre el complejo de Mo-8 hidroxiquinoleína tales como: $\mathrm{NaNO}_{3}-\mathrm{HNO}_{3}, \mathrm{H}_{2} \mathrm{SO}_{4}$ y $\mathrm{KClO}_{3}$. Se decidió trabajar con el sistema Mo-8 hidroxiquinoleína- $\mathrm{KClO}_{3}$, debido a que este sistema es el que aporta la mejor sensibilidad de todos los estudiados, puesto que el $\mathrm{KClO}_{3}$ se comporta como el mejor catalítico de la reacción. Se probaron las técnicas de voltamperometría de despojo catódico, onda cuadrada y barrido lineal. El estudio por voltamperometría cíclica demostró que tanto en el barrido catódico como en el anódico, se presentan señales catódicas. Esto sugirió la posibilidad de que las técnicas normalmente utilizadas en análisis de trazas, a saber, la redisolución catódica con pulso diferencial y la voltamperometría de onda cuadrada, no proporcionaron buenos resultados o la sensibilidad fue baja en comparación con el barrido lineal. Se efectuó por este motivo un estudio utilizando voltamperometría de onda cuadrada, en un equipo BAS $100 \mathrm{~B} / \mathrm{W}$. Este sistema tiene la capacidad de reportar para la onda cuadrada, las corrientes individuales correspondientes a los pulsos catódicos y anódicos. En un sistema normal, sería de esperar que las corrientes fueran de signo contrario, con lo que la diferencia resultante tendría una magnitud mayor que cualquiera de ellas individualmente. Sin embargo, para el sistema de molibdeno, ambas corrientes son positivas. Esto implica que la voltamperometría de onda cuadrada SWV no se recomienda para la determinación de trazas de este elemento. En el caso de la voltamperometría de redisolución con pulso diferencial DPSV no se utilizó 
porque proporcionó corrientes más bajas que las obtenidas con el barrido lineal LSV y las recuperaciones en agua fueron más altas, las cuales fueron cercanas a $110 \%$, mientras que con barrido lineal son cercanas al 100\% óptimo.
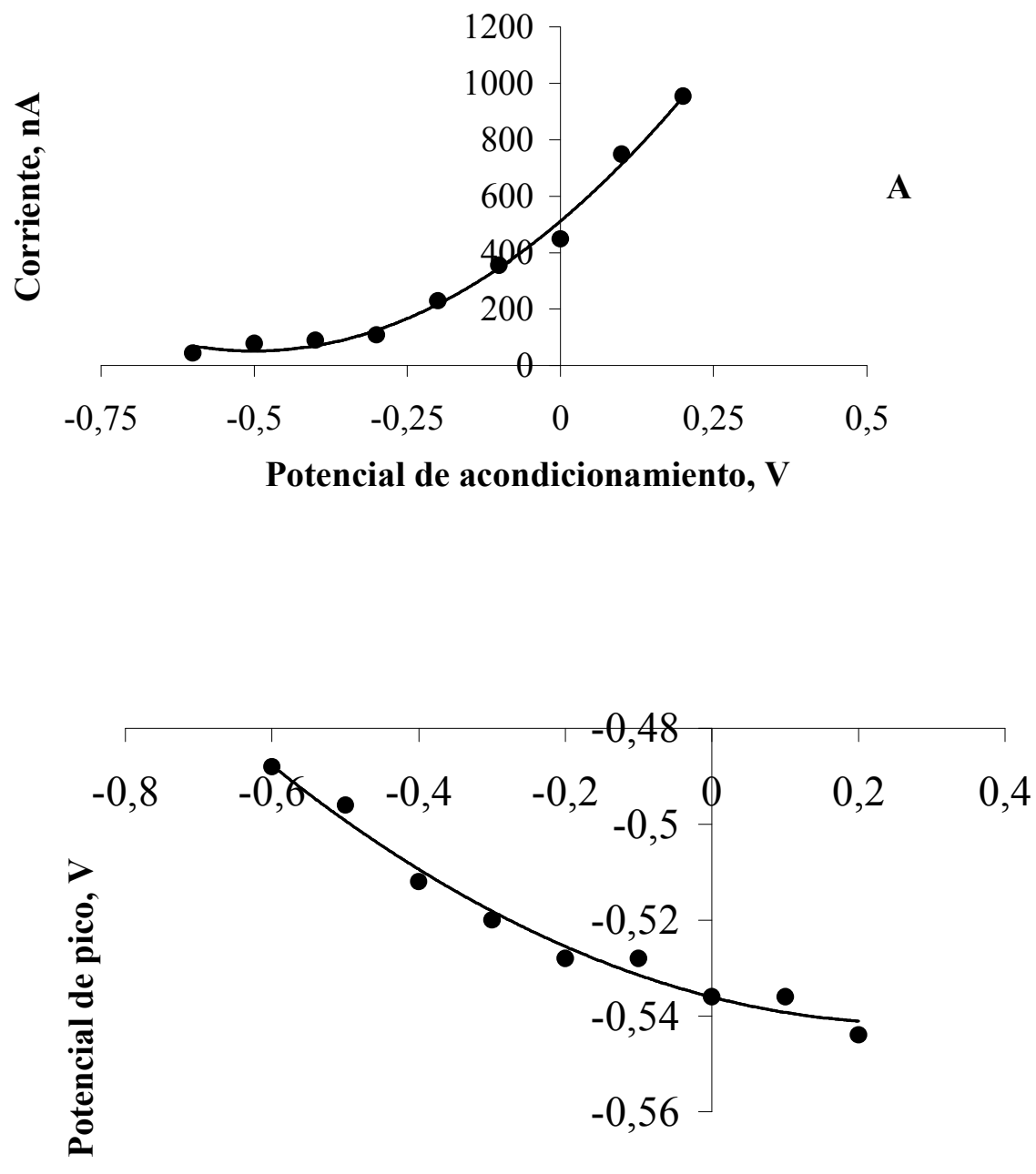

Potencial de acondicionamiento, $\mathrm{V}$

Figura 1. Efecto de potencial de acondicionamiento sobre (A) la corriente de pico de Mo $0.52 \mu \mathrm{g} / \mathrm{L}$ y sobre el potencial del pico de Mo $0.52 \mu \mathrm{g} / \mathrm{L}$. 

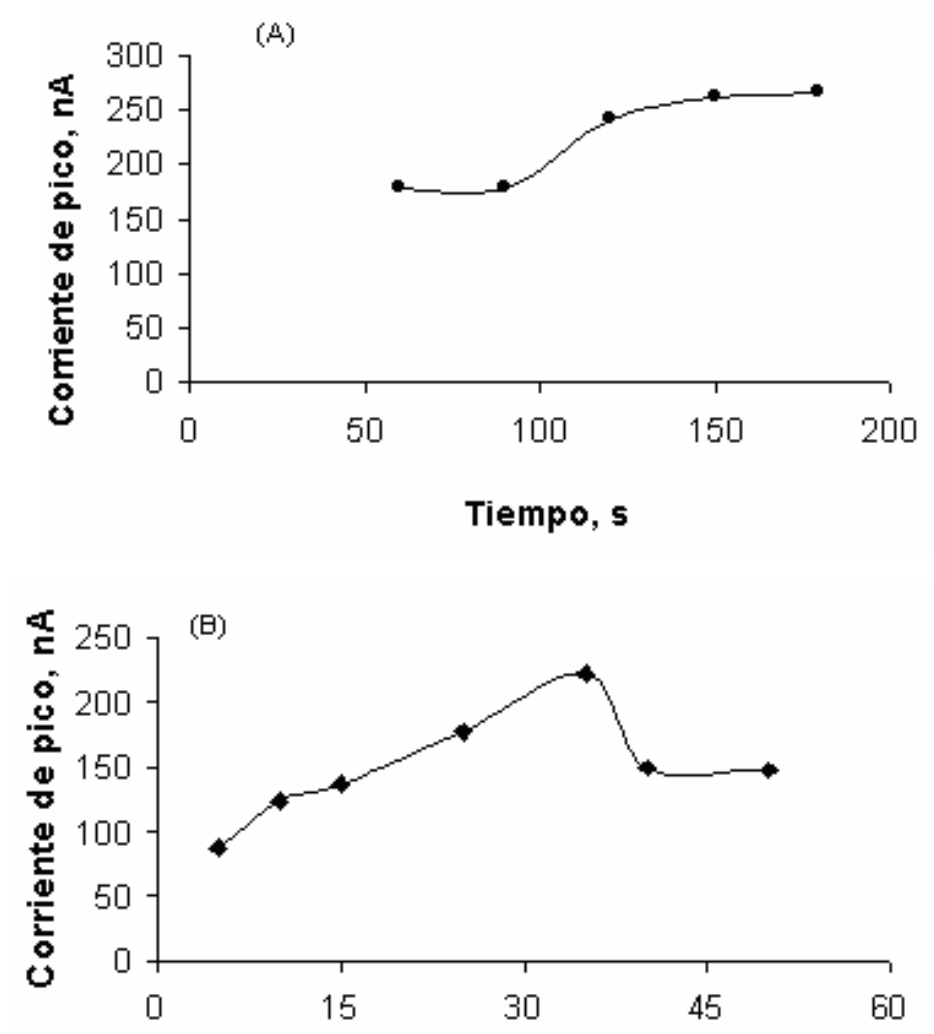

Velocidad, mVis

Figura 2. (A) Efecto del tiempo de electrodepósito sobre la corriente de pico de Mo $0.10 \mu \mathrm{g} / \mathrm{L}$; (B) efecto de la velocidad de barrido sobre la corriente de pico de Mo 0.10 $\mu \mathrm{g} / \mathrm{L}$.

Para optimizar las mejores condiciones de análisis se tomaron como base parámetros recomendados por Gao y Siow [1,8] y Navratilova y Kopanica [4], optimizando un parámetro a la vez. Por ejemplo, en la Fig. 1A se observa el cambio que sufre la corriente de pico del Mo(VI) conforme el potencial de acondicionamiento se hace más negativo, se escogió el potencial de $0.20 \mathrm{~V}$ como el que presenta la señal más sensible.

Es importante observar en la Fig. 1B que el pico de $\mathrm{Mo}(\mathrm{VI}) 0.52 \mu \mathrm{g} / \mathrm{L}$ se va desplazando hacia potenciales más positivos conforme el potencial de acondicionamiento se hace más negativo. Se buscó el mejor tiempo de depósito entre 50 segundos y 200 segundos, se considera que después de 120 segundos la señal no mejora por lo que un tiempo de 90 segundos es suficiente, puesto que el método resultó muy sensible y no se hace necesario electrodepositar por períodos más largos. Esto se puede ver en la Fig. 2A; también en la Fig. 2B se tiene la variación de la señal de Mo con la velocidad de barrido: una velocidad de 40 $\mathrm{mV} / \mathrm{s}$ es suficiente. 

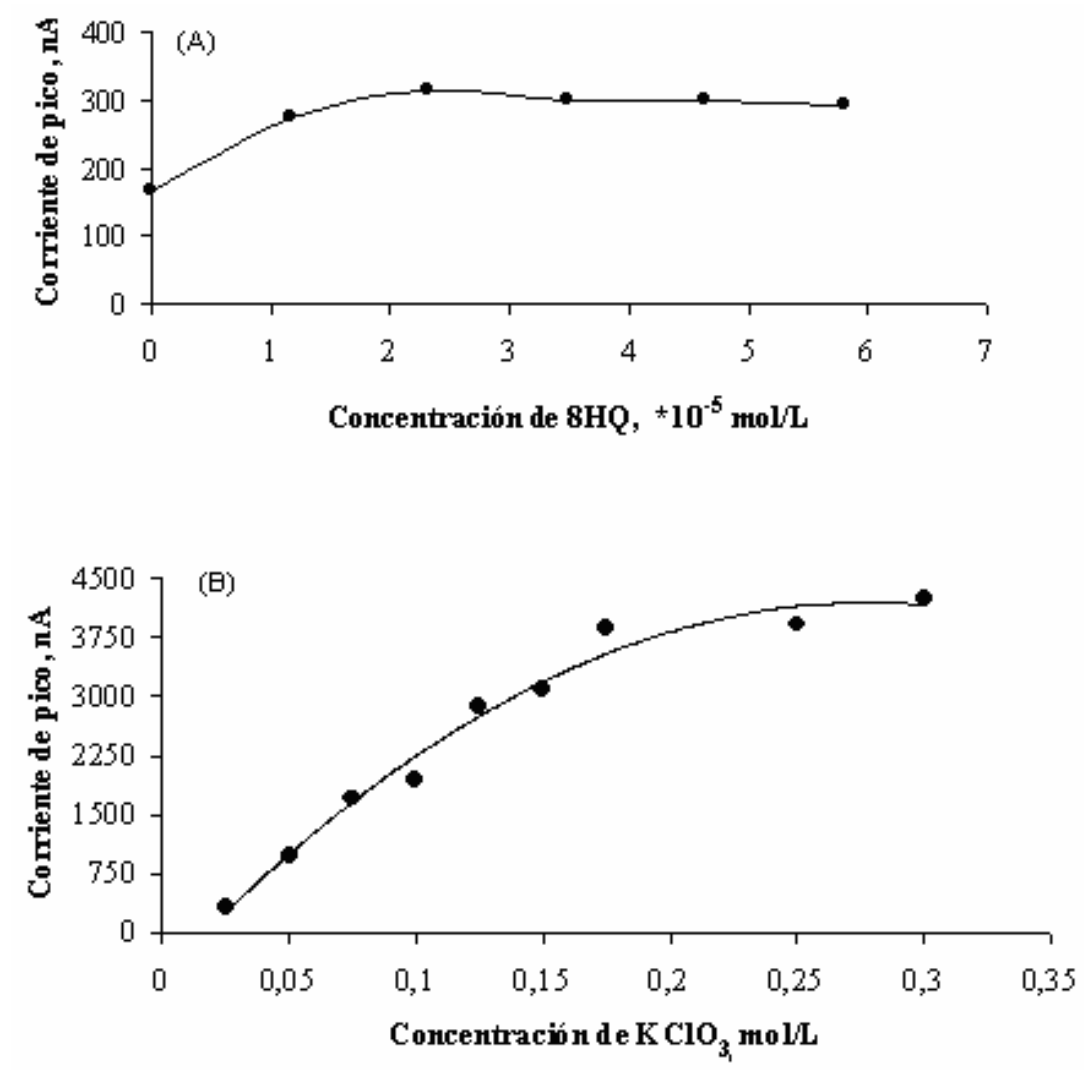

Figura 3. (A) Efecto de la concentración del ligando 8 hidroxiquinoleína sobre la señal de Mo $0.20 \mu \mathrm{g} / \mathrm{L}$; (B) efecto de la señal del agente catalítico $\mathrm{KClO}_{3}$, sobre la señal de Mo $0.20 \mu \mathrm{g} / \mathrm{L}$.

También se estudiaron las concentraciones adecuadas de ligando y catalítico. El ligando se trabajó en concentraciones entre $10^{-3}$ a $10^{-6} \mathrm{~mol} / \mathrm{L}$ de 8 hidroxiquinoleína y la señal se mantiene casi constante entre $10^{-4}$ y $10^{-6} \mathrm{~mol} / \mathrm{L}$. Una concentración de 1 a $2 \times 10^{-5} \mathrm{~mol} / \mathrm{L}$ es adecuada, para obtener una buena señal como se indica en la Fig. 3A. La concentración de electrolito $\mathrm{KClO}_{3}$ también se varió entre $10^{-1}$ y $10^{-2} \mathrm{~mol} / \mathrm{L}$, siendo suficiente una concentración en celda de $0.2 \mathrm{~mol} / \mathrm{L}$ a $0.3 \mathrm{~mol} / \mathrm{L}$. La presencia del catalítico es necesaria porque según Gao y Siow [1,8] el Mo(VI) se une a dos moléculas de la oxima y se adsorbe en el electrodo, posteriormente el complejo se reduce en el electrodo de gota de mercurio, y genera $\mathrm{Mo}(\mathrm{V})$ más la oxima, luego se reduce en la superficie del electrodo de mercurio a Mo(IV) no acomplejado. El Mo(IV) se reoxida a $\mathrm{Mo}(\mathrm{V})$, repetidamente contribuyendo a aumentar la corriente de reducción. 


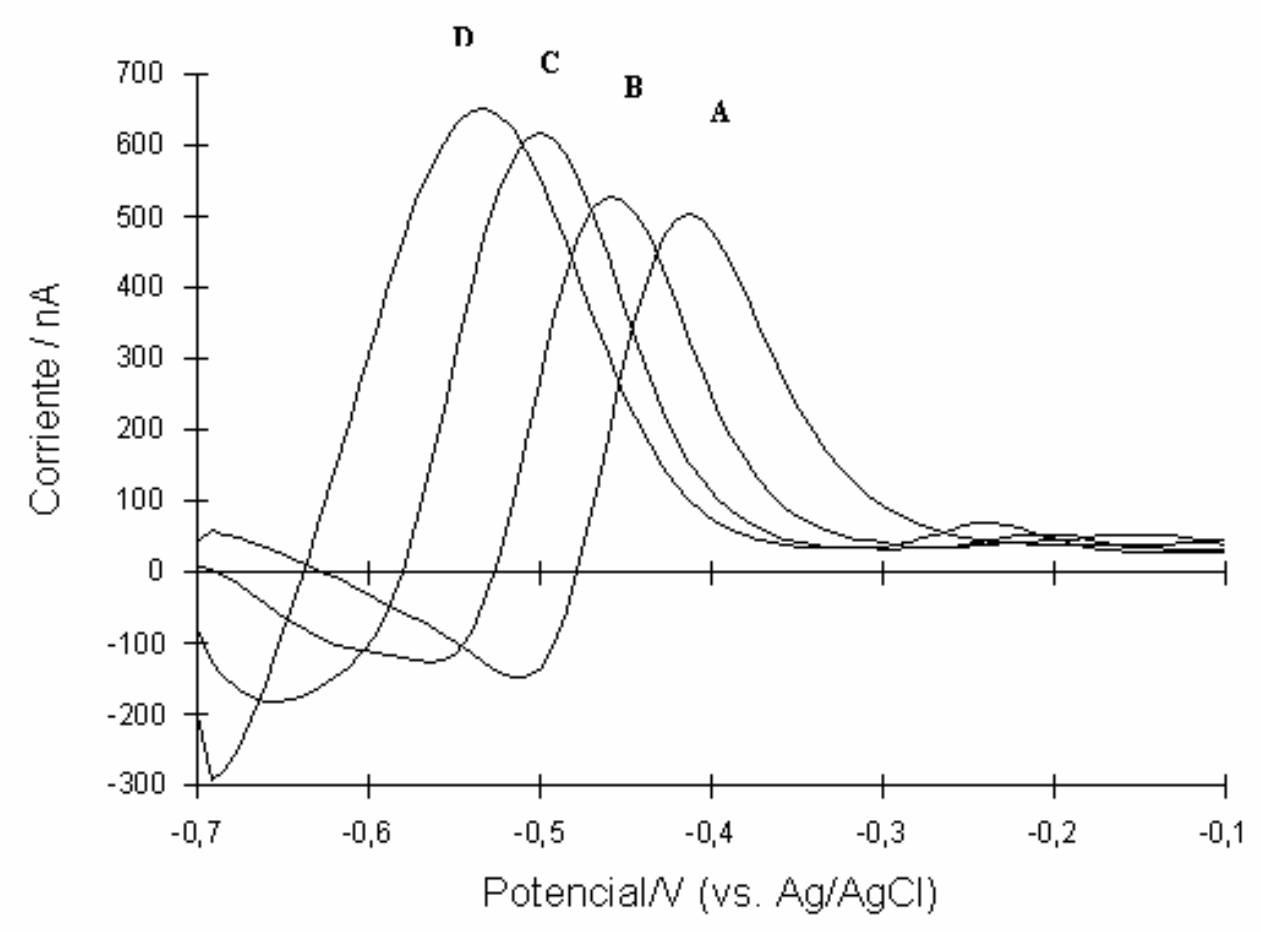

Figura 4. Variación de la altura del pico y corrimiento de la senal para Mo(VI) con el pH. A:3.03, B:3.58, C:4.12, D:4.58.

Un factor importante en la estabilidad de los complejos es el $\mathrm{pH}$; con el fin de obtener señales estables y reproducibles se determinó el $\mathrm{pH}$ que diera la mejor corriente de pico. Se encontró que se puede trabajar a pH entre 3.5 y 4.5 sin que varíe la sensibilidad en forma considerable, lo que si ocurre es que el pico se hace más ancho. Es de notar en la Fig. 4 que conforme se aumenta el pH, se da un desplazamiento del pico de Mo hacia potenciales más negativos con un aumento en la señal del pico de Mo(VI).

El electrolito soporte que se consideró más adecuado está constituido en la celda polarográfica por: $3.5 \mathrm{~mL}$ de disolución de $\mathrm{KClO}_{3} 0.5 \mathrm{~mol} / \mathrm{L}$ y $3.0 \mathrm{~mL}$ de buffer de $\mathrm{CH}_{3} \mathrm{COOH} / \mathrm{CH}_{3} \mathrm{COONa}$ entre $0.2 \mathrm{~mol} / \mathrm{L}$ y $0.4 \mathrm{~mol} / \mathrm{L}$ en acetatos totales, de $\mathrm{pH} 3.5$ pero se puede trabajar hasta un $\mathrm{pH}$ de 4.5 sin que varíe mucho la sensibilidad, además la cantidad óptima de 8-hidroxiquinoleína es $50 \mu \mathrm{L}$ de $1 \times 10^{-2} \mathrm{~mol} / \mathrm{L}$.

Las condiciones optimizadas con la técnica voltamperométrica de barrido lineal se presentan en la Tabla 1.

También se determinó el ámbito lineal, el cual va desde $1.0 \eta \mathrm{g} / \mathrm{L}$ hasta 10 $\mu \mathrm{g} / \mathrm{L}$, con el método resulta adecuado en el ámbito de $\mu \mathrm{g} / \mathrm{L}$ y $\eta \mathrm{g} / \mathrm{L}$. Las linealidades en todos los casos cuentan con un $\mathrm{R}^{2}$ de 0.99 o mayor como se muestra en las Fig. 5A y 5B. A pesar de que el ámbito lineal cubre dos órdenes de magnitud, cambia la sensibilidad al pasar de los $\eta \mathrm{g} / \mathrm{L}$ a $\operatorname{los} \mu \mathrm{g} / \mathrm{L}$ ya que conforme hay más Mo en el medio, más rápido se satura la gota, por lo que se recomienda trabajar en ambas zonas en forma independiente. 
Tabla 1. Condiciones instrumentales para el método de $\mathrm{Mo}-\mathrm{KClO}_{3}$ con $8 \mathrm{HQ}$.

\begin{tabular}{lr}
\hline Técnica & Voltamperometría de barrido lineal LSV \\
\hline Potencial inicial & $-0.200 \mathrm{~V}$ \\
Potencial final & $-0.800 \mathrm{~V}$ \\
Potencial de acondicionamiento & $0.200 \mathrm{~V}$ \\
Tiempo de acondicionamiento & 90 segundos \\
Tiempo de equilibrio & $5 \mathrm{segundos}$ \\
Velocidad de barrido & $40 \mathrm{mV} / \mathrm{s}$ \\
Potencial del pico & $-0.46 \mathrm{~V} \mathrm{a}-0.48 \mathrm{~V}$ \\
\hline
\end{tabular}
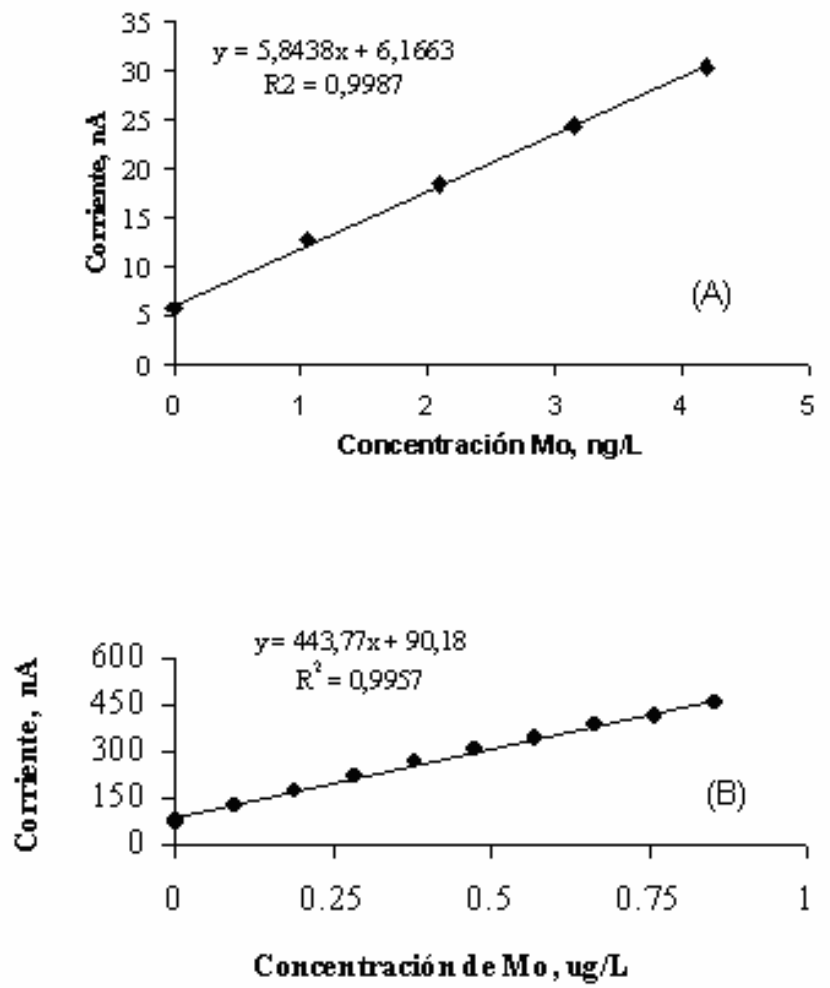

Figura 5. Ámbito lineal para Mo: (A) en el ámbito de los $\eta \mathrm{g} / \mathrm{L}, \mathrm{y}(\mathrm{B})$ en el ámbito de $\mu \mathrm{g} / \mathrm{L}$.

Se determinaron los límites de detección y cuantificación siendo estos de 0.3 $\eta \mathrm{g} / \mathrm{L}$ y de $1.0 \eta \mathrm{g} / \mathrm{L}$, respectivamente, calculados según lo recomienda el método de Taylor, para lo cual se analizó un blanco veinte veces y se determinó la desviación estándar. El límite de detección se consideró como tres veces la desviación estándar del blanco entre la pendiente de calibración y el de cuantificación como diez veces la desviación estándar del blanco entre la pendiente de calibración. 
Se aplicó el método en muestras molidas y liofilizadas de arroz, frijoles, lentejas y garbanzos, cuyos contenidos se detallan en la Tabla 2. Se realizó un estudio para determinar la mezcla digestiva adecuada, la cual resultó ser de 2 $\mathrm{mL}$ de $\mathrm{HNO}_{3}, 250 \mu \mathrm{L}$ de $\mathrm{H}_{2} \mathrm{SO}_{4}$ y $100 \mu \mathrm{L}$ de $\mathrm{HClO}_{4}$; todos los reactivos fueron de calidad ultrapura, para digerir $0.2500 \mathrm{~g}$ de muestra de cada grano molido.

Tabla 2. Contenido de Mo en diferentes granos.

\begin{tabular}{lccc}
\hline \multicolumn{1}{c}{ Matriz } & Contenido en mg/L & Desv.est. & Coeficiente de variación \% \\
\hline Arroz & 1.5 & 0.6 & 27.6 \\
Frijoles rojos & 3.4 & 0.5 & 16.2 \\
Lentejas & 5.9 & 0.6 & 1.7 \\
Garbanzos & 6.0 & 1.0 & 16.2 \\
\hline
\end{tabular}

Debido a que la metodología tiene una alta sensibilidad y límites de detección y cuantificación que permiten el análisis de contenidos en el orden de las ultra trazas $(\mu \mathrm{g} / \mathrm{L}$ o $\mathrm{ng} / \mathrm{L})$, fue necesario agregar en celda solo microporciones de las muestras $(50 \mu \mathrm{L})$, lo cual contribuye a los altos coeficientes de variación. Según Horwitz [15], a concentraciones bajas, en el análisis de trazas y ultra trazas, la desviación estándar es alta y la precisión relativamente baja, con coeficientes de variación del $20 \%$ o mayores, por lo que es aceptable tener coeficientes como los obtenidos. Además los resultados de las recuperaciones en garbanzo oscilaron entre $110 \%$ y $120 \%$. Se recomienda mejorar las condiciones de tratamiento de las muestras de granos como liofilización y homogenización para disminuir la variabilidad de los resultados.

\section{Agradecimientos}

Se agradece a la Vicerrectoría de Investigación, a la Escuela de Química y al Centro de Electroquímica y Energía Química (CELEQ) de la Universidad de Costa Rica por todo el apoyo brindado para llevar a cabo esta investigación.

\section{Resumen}

Se desarrolló un método analítico muy sensible, para determinar molibdeno en agua y granos, utilizando la técnica de voltamperometría de barrido lineal, con preconcentración adsortiva de un complejo de $\mathrm{Mo}(\mathrm{VI})$ con 8 hidroxiquinoleína, en un medio de buffer de acetato de $\mathrm{pH}=3.5$ y $\quad \mathrm{KClO}_{3} 0.5 \mathrm{~mol} / \mathrm{L}$ como electrolito soporte. El ámbito lineal del método va desde $1.0 \eta \mathrm{g} / \mathrm{L}$ hasta $10 \mu \mathrm{g} / \mathrm{L}$, con un límite de detección de $0.3 \eta \mathrm{g} / \mathrm{L}$ y de cuantificación de $1.0 \eta \mathrm{g} / \mathrm{L}$. El método resulta adecuado en el ámbito de $\mu \mathrm{g} / \mathrm{L}$ y $\eta \mathrm{g} / \mathrm{L}$. Se aplicó el método en muestras molidas de arroz, frijoles, lentejas y garbanzos y se logró cuantificar las siguientes cantidades: $6.2 \mathrm{mg} / \mathrm{kg}$ en lentejas, $6.0 \mathrm{mg} / \mathrm{kg}$ en garbanzos, $3.2 \mathrm{mg} / \mathrm{kg}$ en frijoles, y $1.7 \mathrm{mg} / \mathrm{kg}$ en arroz.

Palabras claves: voltamperometría de barrido lineal, complejos, análisis de trazas, molibdeno, 8 hidroxiquinoleína, preconcentración adsortiva. 


\section{Referencias}

1. Z. Gao, K.S. Siow, Talanta 43 (1996) 719-726.

2. $\quad$ K. Yokoi, C. van den Berg, Anal. Chim. Acta 257 (1992) 293-299.

3. J.R. Turnlund, W.R. Keyes, G.L. Peiffer, G. Chiang, Am. J. Clin. Nutr. 61 (1995) 1102-1109.

4. Z. Navratilova, M. Kopanica, Anal. Chim. Acta 244 (1991) 193-196.

5. S. Sander, Fresenius J. Anal. Chem. 365(5) (1999) 435.

6. M. Karakaplan, G. Henze, Electroanalysis 5 (1993) 623-625.

7. M. Karakaplan, S. Gucer, G. Henze, Fresenius J. Anal. Chem. 342 (1992) 186-190.

8. Z. Gao, K. Siow, Mikrochimm. Acta 124 (1996) 211-218.

9. P. Farias, A. Ohara, A. Nóbrega, Electroanalysis 6 (1994) 333-339.

10. J.L. Jiang, Q. Gong, M. Liu, Anal. Letters 27 (1994) 1945-1954.

11. A.M. Alí, M.A. Ghandour, S.A. El Shatoury, S.M. Amed, Electroanalysis 12(2) (2000) 155.

12. I.C. Fraga, P.A. Farias, A.K. Ohara, Fresenius J. Anal. Chem. 366(3) (2000) 307.

13. A. Safavi, E. Shams, Anal. Chim. Acta 396(2-3) (1999) 215.

14. J.K. Taylor, Quality Assurance of Chemical Measurements, Lewis Publishers, Inc., USA. (1987) 159-163.

15. W. Horwitz, Anal. Chem. 54 (1982) 67A-76A.

16. W. Horwitz, R. Albert, J. Assoc. Anal. Chem. 68 (1985) 830-838. 